hen (BFH-Urteil vom 28. Juli 2005 - III R 68/04, BFHE 211, 107, BStBI II 2008, 350; Lange in: Hübschmann/Hepp/Spitaler, § 100 FGO Rz 39, mwN).

Da das FG im Streitfall bei seiner Entscheidung allein auf den Zeitpunkt des Übergangs des Betriebsvermögens auf die Klägerin abgestellt hat, war die Vorentscheidung aufzuheben.

\section{4.}

Die Sache ist nicht spruchreif.

a) Das FG hat nicht geprüft, ob die Klägerin durch einen Verkauf von Bildern des $V$ den Tatbestand des § 13a Abs. 5 Nr. 1 ErbStG verwirklicht hat. Sollten die vom FG noch nachzuholenden tatsächlichen Feststellungen ergeben, dass die Klägerin innerhalb der Behaltensfrist des § 13a Abs. 5 ErbStG Bilder des V verkauft hat, liegt eine Veräußerung wesentlicher Betriebsgrundlagen iSd § 13a Abs. 5 Nr. 1 Satz 2 ErbStG vor. Nach der insoweit maßgeblichen ertragsteuerlichen Betrachtung gehören zu den wesentlichen Grundlagen eines Betriebs im Allgemeinen die Betriebsräume, der (bisherige) betriebliche Wirkungskreis (Betätigungsfeld und Kundschaft) und auch der Warenbestand, soweit er nicht in seiner konkreten Zusammensetzung jederzeit wieder kurzfristig beschaffbar ist (BFH-Urteile vom 24. Juni 1976 - IV R 200/72, BFHE 119, 430,
BStBI II 1976, 672; vom 29. November 1988 - VIII R 316/82, BFHE 156,408, BStBI II 1989, 602). Diese Voraussetzungen sind für die von einem freiberuflichen Kunstmaler geschaffenen und zu seinem Nachlass gehörenden Kunstwerke zu bejahen.

Hat die Klägerin innerhalb von fünf Jahren nach dem Erwerb im Nachlass des $V$ befindliche Kunstwerke veräußert, ist der Freibetrag nur in Höhe der Werte rückwirkend weggefallen, mit denen die Werke in die Bemessungsgrundlage der Erbschaftsteuer eingegangen sind. Dies ergibt sich aus der Verwendung des Wortes "soweit" in der Einleitung des § 13a Abs. 5 ErbStG. Eine innerhalb des Zeitraums von fünf Jahren nicht verwirklichte Veräußerungsabsicht spielt keine Rolle.

b) Das FG hat zu Recht keine tatsächlichen Feststellungen zu dem Vorbringen des FA getroffen, die Klägerin habe die Atelierräume des $V$ an dessen 1. Todestag als Museum und Galerie eröffnet. Ein etwaiger Übergang eines bisher freiberuflichen Betriebsvermögens in gewerbliches Betriebsvermögen und eine entsprechende Umqualifizierung der aus dem Betrieb erzielten Einkünfte führen nämlich als sog. Strukturwandel nicht zu einer Betriebsaufgabe (vgl. BFH-Urteile vom 19. Mai 1981 VIII R 143/78, BFHE 133, 396, BStBI II 1981, 665, und in BFHE 168, 405, BStBI II 1993, 36; Jülicher in: Troll/Gebel/Jülicher, ErbStG, $\S 13 a \mathrm{Rz} 275)$. (Gründe von der Redaktion bearbeitet.)

\title{
Herausgabeanspruch bei NS-verfolgungsbedingt abhanden gekommenen Sachen
}

Kammergericht, Urteil vom 28. Januar 2010 - 8 U 56/09

1. Ein Eigentumsverlust des Klägers an den Plakaten kann weder aus der Wegnahme im Auftrag des Reichspropagandaministeriums im Jahre 1938 hergeleitet werden, noch hat ein Übergang in das Volkseigentum in der DDR stattgefunden. Auch durch die Abfindungszahlungen im Wiedergutmachungsverfahren oder aus der Untätigkeit des Klägers resultiert kein Eigentumsverlust an den Plakaten.

2. Die verspätete Geltendmachung der Herausgabeforderung durch den Kläger verstößt gegen Treu und Glauben, selbst wenn sich die Beklagte im Einklang mit den Grundsätzen der Washingtoner Konferenz nicht auf Verjährung beruft. Das Herausgabeverlangen ist verwirkt, wenn über einen bloßen Zeitablauf hinaus besondere Umstände bestehen, die der Durchsetzung des Anspruchs entgegenstehen. (Leitsätze der Redaktion)

- Auf die Berufung der Beklagten wird das am 10.2.2009 verkündete Urteil des Landgerichts Berlin - 190 116/08 [abgedruckt in: KUR 2009, 57 ff.] teilweise geändert und wie folgt neu gefasst: Es wird festgestellt, dass der Kläger nicht berechtigt ist, die im Besitz der Beklagten befindlichen Plakate aus der Plakatsammlung des Zahnarztes Dr. XX (Sammlungszeitraum 1896 bis 1938), die durch einen Aufkleber oder eine Stempelung als von Dr. XX gesammelt identifizierbar sind derzeit identifiziert: 4.259 Plakate - herauszuverlangen. [...]

\section{Gründe:}

I.

Der Kläger verlangt als Erbeserbe Herausgabe von zwei Plakaten aus einer Sammlung, die seinem Vater Dr. XX 1938 im Auftrag des Reichspropagandaministeriums weggenommen wurde und zu einem erheblichen Teil in den Besitz des Museums für Deutsche Geschichte und sodann der Beklagten 
gelangt ist. Die Beklagte begehrt widerklagend die Feststellung, dass der Kläger nicht Eigentümer der in ihrem Besitz befindlichen, durch Aufkleber oder Stempelung als von Dr. XX gesammelten zu identifizierenden Plakate sei, hilfsweise die Feststellung, dass der Kläger nicht Herausgabe dieser Plakate verlangen könne.

Das Landgericht hat die Beklagte zur Herausgabe des Plakates „Dogge“ verurteilt, die Klage hinsichtlich des Plakates "Die blonde Venus" und die Widerklage hat es abgewiesen. [...] Gegen dieses Urteil, welches der Beklagten am 12.2.2009 und dem Kläger am 13.2.2009 zugestellt worden ist, haben beide Parteien am 12.3.2009 Berufung eingelegt. [...]

Mit seiner Berufung verfolgt der Kläger seine Klage auf Herausgabe des Plakates "Die blonde Venus" weiter und macht geltend: Die Zugehörigkeit dieses Plakates zur Sammlung seines Vaters werde entgegen der Auffassung des Landgerichts durch die vorgetragenen Indizien hinreichend belegt, insbesondere weil es - was unstreitig ist - zusammen mit durch Stempel/Aufkleber einwandfrei der Sammlung XX zuzuordnenden Plakaten verpackt aufgefunden und bei Überführung in die Bestände des XX bzw. der Beklagten der Sammlung XX zugeordnet wurde. [...]

Die Zulässigkeit des Rechtsweges sei in der Berufungsinstanz gemäß § 17a Abs. 5 GVG nicht mehr zu prüfen. Ohnehin sei der vorliegende Fall dadurch gekennzeichnet, dass keine Eigentumsentziehung erfolgt sei, sondern ein Raub durch staatliche Organe im Geltungsbereich des Bundesrückerstattungsgesetzes. Nach diesem Gesetz abgearbeitete Fälle unterfielen nicht dem Vermögensgesetz (im Folgenden: VermG). Die erfolgte Wiedergutmachungszahlung schließe den Anspruch auf Rückgabe des Vermögenswertes nicht aus, sondern sei lediglich zurückzuzahlen, wenn die Plakatsammlung wieder in die Verfügungsgewalt des Klägers gelangt sei. Hinsichtlich des Verwirkungseinwandes bedürfe es für das Vertrauensschutzmoment mehr als der bloßen Nutzung der Plakate.

Die Beklagte wendet sich mit ihrer Berufung gegen ihre Verurteilung zur Herausgabe des Plakates "Dogge“ und gegen die Abweisung der Widerklage und macht geltend: Die Klage sei als unzulässig abzuweisen, weil der Restitutionstatbestand des $§ 1$ Abs. 6 VermG Vorrang genieße; eine Verweisung an das Verwaltungsgericht scheide mangels eines fristgerechten Restitutionsantrages des Klägers aus und deshalb sei § 17a Abs. 5 GVG nicht anwendbar. Für die Anwendbarkeit des VermG komme es nach der Rechtsprechung des Bundesverwaltungsgerichts und des Bundesgerichtshofs nicht darauf an, ob die NS-Unrechtsmaßnahme zu einem Verlust des Eigentums geführt habe. Jedenfalls sei ein Herausgabeanspruch des Klägers aufgrund des Vorranges der Wiedergutmachungsregeln des alliierten Rückerstattungsrechts und des Bundesrückerstattungsgesetzes ausgeschlossen.
Aufgrund des Berichts von Dr. XX aus dem Jahre 1953 und der Erklärung des Dr. XX vom 28.11.1946 sei von einer Übertragung des Eigentums an der Plakatsammlung unter Vereinbarung eines Besitzkonstituts - und nicht nur zum Schein - auszugehen. Durch die - wenn auch rechtsstaatswidrige - Konfiskation im Jahre 1938 sei Reichseigentum entstanden. In der DDR seien die Plakate Eigentum des Volkes geworden. Ansonsten liege im Brief des Dr. XX an Herrn XX vom 23.5.1966 eine Aufgabe des Eigentums.

Die Beklagte, welche die Einrede der Verjährung ausdrücklich nicht erhebt, beruft sich auf Verwirkung, weil, obwohl Dr. XX seit 1966 vom Verbleib der streitgegenständlichen Teile der Plakatsammlung wusste, weder er noch - trotz der Frist des VermG zum 30.6.1993 - seine Witwe noch der Kläger selbst bis 2006 Herausgabe verlangten und weil sich das Museum für Deutsche Geschichte bzw. nachfolgend die Beklagte auf die Erklärung von Dr. XX, im Hinblick auf die erhaltene Entschädigung keinen Anspruch an seinem früheren Besitz geltend machen zu wollen, verlassen und eingerichtet hätten, indem die Plakate in die eigene wesentlich umfangreichere Plakatsammlung integriert, bewahrt und erschlossen wurden. [...]

\section{II.}

Die zulässige Berufung der Beklagten hat zum Teil Erfolg, während die zulässige Berufung des Klägers unbegründet ist. Die Klage ist zulässig, aber unbegründet. Die zulässige Widerklage hat allein mit dem Hilfsantrag Erfolg.

1. Die Klage ist zulässig. [...] § 17a Abs. 5 GVG ist nicht wie die Beklagte meint - deshalb unanwendbar, weil in vermögensrechtlichen Angelegenheiten keine Verweisung von der ordentlichen Gerichtsbarkeit an das Verwaltungsgericht in Betracht kommt (BGH NJW 1993, 332, 333) und deshalb keine Fortsetzung des Rechtsstreits in (ggf. mehreren Instanzen) einer weiteren Gerichtsbarkeit droht. Eine derartige einschränkende Auslegung findet im Wortlaut des § 17a GVG keine Grundlage und ist auch nach dem Sinn und Zweck der Regelung, eine zügige Sachentscheidung zu fördern, nicht geboten.

2. Die Klage ist jedoch unbegründet.

a. Ein Herausgabeanspruch scheidet im Hinblick auf den Vorrang des alliierten Rückerstattungsrechts und der Wiedergutmachungsvorschriften des Bundesrückerstattungsgesetzes aus.

Ansprüche des Vaters des Klägers auf Rückgabe der Plakatsammlung - auch gegenüber dritten Erwerbern und Besitzern - fielen unter Art. 1 der Anordnung BK/O (49) 180 der Alliierten Kommandantur Berlin über die Rückerstattung feststellbarer Vermögensgegenstände an Opfer der nationalsozialistischen Unterdrückungsmaßnahmen vom 26.7.1949 
(Verordnungsblatt für Groß-Berlin, Teil I, Seite 221), weil ihm die Sammlung aus Gründen der Rasse - wegen jüdischer Abstammung - ungerechtfertigt entzogen worden war. Ansprüche, die unter diese Anordnung fielen, konnten gemäß Art. 51 Satz 1 grundsätzlich nur in dem Verfahren nach dieser Anordnung geltend gemacht werden. Ferner ist durch $\S 7$ Bundesrückerstattungsgesetz festgeschrieben, dass rückerstattungsrechtliche Ansprüche nur nach Maßgabe dieses Gesetzes, d.h. im Rückerstattungsverfahren geltend zu machen sind.

Es entspricht auch der gefestigten höchstrichterlichen Rechtsprechung, dass Ansprüche von Betroffenen nationalsozialistischer Unrechtsakte nur nach Maßgabe der Rückerstattungs- und Entschädigungsgesetze geltend gemacht werden können (BGHZ 9, 34; BGH RzW 1956, 237) bzw. dass die Regelung, die in den Rückerstattungsgesetzen der einzelnen Besatzungszonen getroffen worden ist, eine Rückforderung nach allgemeinem bürgerlichen Recht wegen Entziehungsvorgängen ausschließt, die sich im Rahmen der allgemeinen Verfolgungsmaßnahmen gehalten haben (BGHZ 10, 340). Die alliierten Rückerstattungsgesetze haben sich ausschließliche Geltung für alle in Betracht kommenden Rückerstattungsansprüche zugemessen, unabhängig von der etwaigen Nichtigkeit der Entziehungsmaßnahme; im ordentlichen Rechtsweg durften Ansprüche auf Herausgabe von Vermögenswerten nur geltend gemacht werden, wenn sie nicht auf verfolgungsbedingte Gründe gestützt waren (BVerwGE 98, 261). Dementsprechend ist auch den Restitutionstatbeständen des Vermögensgesetzes Vorrang vor zivilrechtlichen Ansprüchen zuerkannt worden (BVerwG aaO; BGHZ 130, 231)

Der erkennende Senat schließt sich dieser Auffassung an. Der Vorrang, welcher dem Rückerstattungsrecht - aus gutem Grund, nämlich zur geordneten Entwirrung der durch nationalsozialistische Unrechtsakte geschaffenen Fakten - eingeräumt worden ist, ist zu beachten, und zwar auch dann, wenn es wie im vorliegenden Fall nicht zu einer förmlichen Enteignung gekommen ist. Ein Herausgabeanspruch gegenüber der Beklagten ist damit ausgeschlossen, denn einen Rechtsgrund, der mit der nationalsozialistischen Verfolgung - und deshalb mit dem Rückerstattungs- und Wiedergutmachungsrecht - nichts zu tun hätte, macht der Kläger nicht geltend. Dass sich ein Berechtigter nach dem VermG nicht mit früher gewährten Ausgleichsleistungen begnügen muss (vgl. BVerwG ZIP 1997, 1392 und § 7a Abs. 2 VermG), betrifft nur das Verhältnis der Rückerstattungsnormen untereinander, lässt aber keinen bürgerlich-rechtlichen Anspruch auf Naturalrestitution aufleben.

Die "Grundsätze der Washingtoner Konferenz in Bezug auf Kunstwerke, die von den Nationalsozialisten beschlagnahmt wurden" vom 3.12.1998 und die „Erklärung der Bundesregierung, der Länder und der kommunalen Spitzenverbände zur Auffindung und zur Rückgabe NS-verfolgungsbedingt entzo- genen Kulturgutes, insbesondere aus jüdischem Besitz" vom Dezember 1999 ändern daran nichts. Auch der Kläger macht nicht geltend, dass es sich hierbei um Normen handeln würde, aus denen sich ein Rechtsanspruch ableiten ließe.

b. Die Frage, ob ein Herausgabeanspruch des Klägers außerdem im Hinblick auf den bereits erwähnten Vorrang des VermG ausgeschlossen ist, kann deshalb dahinstehen und sei hier nur angerissen:

Die Voraussetzungen nach dem Wortlaut des § 1 Abs. 6 VermG sind erfüllt, denn es geht um einen vermögensrechtlichen Anspruch eines Bürgers jüdischer Abstammung (bzw. dessen Rechtsnachfolgers), der in der Zeit vom 30.1.1933 bis 8.5.1945 aus rassischen Gründen verfolgt wurde und deshalb sein Vermögen infolge Enteignung oder auf andere Weise verloren hat. Es kommt nicht darauf an, dass der Vater des Klägers nicht förmlich enteignet wurde, da § 1 Abs. 6 VermG nach seinem Wortlaut und gefestigter höchstrichterlicher Rechtsprechung auch faktische Vermögensentziehungen erfasst (BVerwGE 98, 261; BVerwG Buchholz 428 § 1 VermG Nr 100; BVerwG VIZ 2000, 284; BGHZ 153, 258 = VIZ 2003, 179). Den Entscheidungen BGH VIZ 1996, 87, BGH ZOV 2003, 322 und KG ZOV 2003, 104 ist - entgegen dem angefochtenen Urteil - nichts Abweichendes zu entnehmen, denn sie betreffen nicht § 1 Abs. 6 VermG, sondern die Frage, ob Vermögensgegenstände im Sinne von § 1 Abs. 3 VermG aufgrund unlauterer Machenschaften „erworben“ worden sind.

Der für eine Anwendung des (von der Volkskammer der DDR erlassenen) VermG erforderliche Bezug des Vermögensverlustes zum Beitrittsgebiet (BVerwG VIZ 2000, 719) dürfte aber fehlen, weil die Plakate in Berlin-Schöneberg - im späteren Westteil Berlins - weggenommen wurden. Dass sie sodann nach Berlin-Mitte - ins spätere Beitrittsgebiet - gelangten, in dem eine Wiedergutmachung nationalsozialistischen Unrechts weithin nicht erfolgte und wodurch eine Rückübertragung (in Natur) nach alliiertem und bundesdeutschem Rückerstattungsrecht faktisch unmöglich war, vermag dem Urteil des Bundesverwaltungsgerichts vom 25.11.2009-8 C 12/08 - zufolge keine Anwendung des VermG zu rechtfertigen, weil das VermG auf Schädigungen im Beitrittsgebiet und nicht auf Nachbesserung von Entscheidungen nach alliiertem Rückerstattungsrecht oder Wiedergutmachungsrecht der Bundesrepublik Deutschland zielt. Diese Sichtweise erscheint auch mit dem Beschluss des Bundesverfassungsgerichts vom 6.2.2004 (VIZ 2004, 220) im Einklang.

c. Die Klage ist ferner im Hinblick auf Treu und Glauben (§ 242 BGB) abzuweisen, weil Herausgabeansprüche des Klägers verwirkt sind.

Ein Recht ist verwirkt, wenn der Berechtigte es längere Zeit hindurch nicht geltend gemacht hat und der Verpflichtete sich darauf eingerichtet hat und nach dem gesamten Verhalten des Berechtigten auch darauf einrichten durfte, dass 
dieser das Recht nicht mehr geltend machen werde (BGH NJW 2006, 219). Der Zeitablauf allein reicht nicht aus, um Verwirkung anzunehmen. Es fällt aber im vorliegenden Fall doch erheblich ins Gewicht, dass eine Herausgabe der Plakate erst im Jahre 2006 verlangt worden ist, obwohl der Vater des Klägers schon 40 Jahre zuvor um den Verbleib erheblicher Teile seiner Plakatsammlung im Museum für Deutsche Geschichte wusste. Wegen der Undurchsetzbarkeit eines Herausgabeanspruchs zu Zeiten der DDR könnte zwar nicht allein auf den Zeitablauf bis 1990 abgestellt werden; dieser Zeitraum ist aber nach dem Rechtsgedanken des § 206 BGB (bzw. § 203 Abs. 2 BGB a. F.) mit zu berücksichtigen. Hinzu kommt vor allem der Inhalt des Schreibens von Dr. XX vom 23.5.1966 an den Mitarbeiter XX des Museums für Deutsche Geschichte, wonach er lediglich ideell und nicht materiell an einer Zusammenarbeit interessiert sei und schon vor einiger Zeit durch einen deutschen Gerichtsbeschluss eine größere Abfindungssumme ausgezahlt bekommen habe, die alle seine Ansprüche gedeckt habe. In die gleiche Richtung weist der im Winter 1970/71 erschienene Artikel von Dr. XX zum Auftauchen seiner Plakatsammlung (und einer weiteren Sammlung in Köln), in dem es u. a. heißt: „Eine vierzigjährige Sammeltätigkeit (...) hat sich als gerechtfertigt erwiesen. West- und Ostdeutschland werden, dessen bin ich sicher, ihre Schätze zu hüten wissen". Auf einen hierdurch zum Ausdruck gebrachten Willen des Sammlers hat bereits die "Beratende Kommission für die Rückgabe NS-verfolgungsbedingt entzogener Kulturgüter" ihre Empfehlung vom 25.1.2007 gestützt, die Sammlung im Deutschen Historischen Museum zu belassen. Auch wenn man bei den Aussagen von Dr. XX die damalige Aussichtslosigkeit eines Herausgabeverlangens mit berücksichtigt (s.a. OLG Brandenburg OLG-NL 1995, 135), durfte die Beklagte darauf vertrauen, die Plakate aus der Sammlung XX auf Dauer behalten zu dürfen, nachdem lange über die Wiedervereinigung hinaus keine Rückgabe verlangt worden war. Eine solche Vertrauensabstimmung ist aus Sicht des Senats bereits mit dem Ablauf der Frist des § 30a Abs. 1 Satz 1 VermG zum 30.6.1993 entstanden, weil es nahe gelegen hätte, vermögensrechtliche Ansprüche anzumelden; eine Anwendbarkeit von § 1 Abs. 6 VermG auf Vermögensgegenstände, die erst nach der Schädigung ins Beitrittsgebiet gelangten, kam immerhin ernsthaft in Betracht (bejahend: VG Berlin ZOV 2008, 115; Neuhaus in: Fieberg/Reichenbach/ Messerschmidt/Neuhaus, VermG, § 1 Rn. 134), auch wenn sie in der Entscheidung des Bundesverwaltungsgerichts vom 25.11.2009 nunmehr verneint worden ist. Erst recht konnte die Beklagte nach Ablauf von weiteren 12 Jahren darauf vertrauen, nicht mehr auf Herausgabe in Anspruch genommen zu werden. Gerade angesichts der genannten Äußerungen von Dr. XX brauchte die Beklagte schwerlich damit zu rechnen, dass seine nächsten Angehörigen und Erben (womöglich) nur deshalb untätig blieben, weil sie nicht um den Verbleib der Plakate wussten. Die Beklagte hat sich auch tatsächlich darauf eingerichtet, keinem Herausgabeverlangen mehr ausgesetzt zu werden. Sie hat die Plakate in die eigene Sammlung integriert, bewährt und erschlossen sowie u. a. im Sommer 1992 - unter besonderer Würdigung von Dr. XX zum Kern einer Ausstellung gemacht. Für die jahrzehntelange geordnete Bewahrung von über 4.000 Plakaten ist von einem erheblichen Aufwand des Museums auszugehen, der zumindest im Einzelfall („Die blonde Venus“) Restaurierungsarbeiten mit einschloss. Dies stellt sich nicht, wie der Kläger meint, als bloße Nutzung dar, sondern als Betätigung des Vertrauens der Beklagten, die Plakate im eigenen Bestand zu behalten. Die verspätete Geltendmachung der Herausgabeforderung verstößt damit gegen Treu und Glauben (s.a. BGH NJW 2003, 824 mit weiteren Nachweisen). Daran ändert nichts, dass sich die Beklagte - offenbar im Hinblick auf die Grundsätze der Washingtoner Konferenz - nicht auf Verjährung, also einen bloßen Zeitablauf beruft, denn im Rahmen der Verwirkung geht es gerade darum, ob darüber hinaus besondere Umstände bestehen, die der Durchsetzung eines Anspruchs entgegenstehen.

\section{$[\ldots]$}

3. Die Widerklage ist gemäß § 33 ZPO zulässig, mit ihrem Hauptantrag, das fehlende Eigentum des Klägers festzustellen, jedoch unbegründet.

a. Es kann nicht festgestellt werden, dass der Vater des Klägers sein Eigentum an der Plakatsammlung vor deren Wegnahme eingebüßt hätte. Von einer Veräußerung an Dr. $X X$ ist aus den im Wesentlichen zutreffenden Gründen des angefochtenen Urteils nicht auszugehen. Auch wenn eine Übereignung aufgrund der Darstellungen des Dr. XX („förmlich übereignet") und des Dr. XX („übereignete ... als Pfand“) als - gemäß § 930 BGB - möglich erscheint und nicht auszuschließen ist, dass es noch zur offenbar vereinbarten Begutachtung durch einen Sachverständigen kam, ist die Vereinbarung eines konkreten Besitzmittlungsverhältnisses nicht hinreichend dargetan. Das Landgericht hat zu Recht darauf hingewiesen, dass es für einen Sicherungsvertrag bereits an einer zu sichernden Forderung, fehlte. Im Rahmen eines Treuhandverhältnisses hätte vielmehr eine Übergabe an Dr. $X X$ als Treuhänder nahe gelegen. Im Übrigen ist mit dem angefochtenen Urteil von einem (gemäß § 117 BGB unwirksamen) Scheingeschäft auszugehen, weil es den Beteiligten offenbar nur darum ging, eine Beschlagnahme der Sammlung zu vermeiden. Dieser Erfolg hing nicht von einer Gültigkeit der Übereignung ab - was ein Scheingeschäft ausschließen würde (vgl. Ellenberger in: Palandt, BGB, 68. Auflage, § 117 Rn. 4 mit weiteren Nachweisen) -, sondern nur vom äußeren Anschein gegenüber den Behörden. Es spricht nichts dafür, dass Dr. XX das Recht eingeräumt werden sollte, gemäß § 903 BGB mit den Plakaten nach seinem Belieben zu verfahren.

b. Ferner hat das Landgericht zutreffend ausgeführt, dass Dr. XX sein Eigentum an den Plakaten nicht durch deren Wegnahme im Jahre 1938 verloren hat, schon weil es an einem Enteignungsakt nach damaliger Rechtslage fehlte. Die Argumentation im Schriftsatz der Beklagten vom 28.1.2010, 
es sei gleichwohl Reichseigentum entstanden, wird durch die dort angeführten Entscheidungen nicht gestützt. Das Urteil BVerwGE 98, 261, geht vielmehr davon aus, dass der durch die 11. Verordnung zum Reichsbürgergesetz vom 25.11.1941 angeordnete Vermögensverfall unwirksam war und nicht zum zivilrechtlichen Verlust des Eigentums führte (aaO Tz. 10), und stellt für den Ausschluss zivilrechtlicher Ansprüche durch die Rückerstattungsgesetze entscheidend darauf ab, "dass die Entziehungsmaßnahme dem Reich zumindest den Schein des Eigentums verschafft und den Vermögensgegenstand dem Verfolgten tatsächlich entzogen hatte" (aaO Tz. 22). Soweit im Urteil BGHZ 153, 258, eine Anordnung der kommissarischen Verwaltung nach der Verordnung über die Behandlung von Vermögen der Angehörigen des ehemaligen polnischen Staates vom 17.9.1940 als Entziehung des Eigentums gewertet worden ist, lässt sich dies nicht auf den vorliegenden Fall übertragen, in dem die Vermögensgegenstände ohne jeglichen Rechtsakt lediglich faktisch entzogen wurden.

c. Es ist nicht ersichtlich, dass die streitgegenständlichen Plakate zu DDR-Zeiten in Volkseigentum übergegangen wären. Einen entsprechenden Rechtsakt hat die Beklagte nicht dargetan. Dass die Plakate, nachdem sie 1953 aufgefunden worden waren, zum Bestand des Museums für Deutsche Geschichte genommen wurden, reicht nicht aus. Soweit sich die Beklagte darauf beruft, dass das Vermögen des früheren Deutschen Reiches durch die Anordnung Nr. 54 der Ministerien des Innern und der Finanzen vom 1.10.1950 auf die Organe und Behörden der DDR übertragen wurde, ist dies für den vorliegenden Fall schon deshalb unerheblich, weil es sich bei der Sammlung - wie erörtert - nicht um Reichsvermögen handelte.

d. Ebenso wenig hat Dr. XX sein Eigentum durch den Vergleich vom 7.3.1961 im Wiedergutmachungsverfahren eingebüßt. Dazu kann auf die Ausführungen im angefochtenen Urteil verwiesen werden. Soweit die Beklagte in der mündlichen Verhandlung vor dem Senat die Meinung vertreten hat, mit diesem Vergleich bzw. mit der Zahlung der vereinbarten Entschädigung in Höhe von 225.000 DM sei das Eigentum von Dr. XX auf die Bundesrepublik Deutschland übergegangen, findet sich hierfür weder in der Anordnung der Alliierten Kommandantur BK/O (49) 180 noch im Bundesrückerstattungsgesetz eine Grundlage und es ist auch keinerlei Rechtsprechung in diesem Sinne ersichtlich. Gegen einen solchen Eigentumsübergang spricht vielmehr § 7a Abs. 2 VermG, wonach Ausgleichsleistungen einem Restitutionsanspruch des vom Vermögensverlust Betroffenen nicht entgegenstehen, sondern lediglich im Falle der Restitution herauszugeben sind (s.a. BVerwG ZIP 1997, 1392).

e. Das Landgericht hat auch zutreffend begründet, weshalb sich aus dem Schreiben des Vaters des Klägers vom 23.5.1996 an Herrn XX weder eine Aufgabe des Eigentums im Sinne von § 959 BGB noch eine Übereignung herleiten lässt; konkrete Argumente hiergegen bringt die Berufungsbegründung der Beklagten nicht vor. Dass aus der jahrelangen Untätigkeit des Klägers und seiner Mutter kein Eigentumsverlust hergeleitet werden kann, stellt auch die Beklagte in zweiter Instanz zu Recht nicht mehr in Frage.

4. Hinsichtlich des Hilfsantrages auf Feststellung, dass der Kläger keine Herausgabe der Plakate beanspruchen kann, ist die Widerklage gemäß den Ausführungen zu II. 2.a und II.2.C begründet. [...] (Gründe von der Redaktion bearbeitet.) 